\title{
Análise dialélica de linhagens de milho com ênfase na resistência a doenças foliares
}

\author{
Thiago Hideyo Nihei(1) e Josué Maldonado Ferreira(1)
} (1)Universidade Estadual de Londrina, Departamento de Ciências Biológicas, Caixa Postal 6001, CEP $86051-990$ Londrina, PR.
E-mail: thiagonihei@hotmail.com, josuemf@uel.br

Resumo - O objetivo deste trabalho foi identificar o tipo de ação gênica predominante para resistência a Exserohilum turcicum, Phaeosphaeria maydis, Physopella zeae e Puccinia polysora, e determinar o potencial genético de linhagens endogâmicas de milho (Zea mays) para a obtenção de híbridos com elevado desempenho agronômico e resistência a doenças foliares. Os 41 híbridos $\mathrm{F}_{1}$, provenientes de cruzamentos dialélicos entre dez linhagens endogâmicas, e as testemunhas P3069, P30F90, BG7060, Balu761 e Dow2A120 foram avaliados em quatro locais, tendo-se utilizado o delineamento experimental de blocos ao acaso, com três repetições. Os híbridos $\mathrm{LGS}_{3} \mathrm{xLGS}$, $\mathrm{LGS}_{2} \mathrm{xLGS}, \mathrm{LGS}_{2} \mathrm{xLGS}$ e $\mathrm{LGS}_{2} \mathrm{xLGS}$ apresentaram excelente desempenho em comparação às testemunhas, quanto aos diferentes caracteres avaliados. As linhagens com maior frequência de alelos favoráveis foram $\mathrm{LGS}_{2}, \mathrm{LGS}_{9}, \mathrm{LGS}_{4}$ e $\mathrm{LGS}_{3}$. Os efeitos gênicos aditivos são os mais importantes para a resistência a $P$. maydis e altura de espiga, enquanto os não aditivos são mais importantes para a produtividade, altura de planta, resistência à E. turcicum, $P$. zeae e $P$. polysora.

Termos para indexação: Exserohilum turcicum, Phaeosphaeria maydis, Physopella zeae, Puccinia polysora, Zea mays, efeito genético.

\section{Diallel analysis of maize inbred lines with emphasis on resistance to leaf diseases}

\begin{abstract}
The objective of this work was to identify the predominant type of gene action for resistance to Exserohilum turcicum, Phaeosphaeria maydis, Physopella zeae and Puccinia polysora, and to determine the genetic potential of maize (Zea mays) inbred lines in order to obtain hybrids with high agronomic performance and resistance to leaf diseases. Forty-one $\mathrm{F}_{1}$ hybrids from the diallel crosses between ten endogamic lines, and the controls P3069, P30F90, BG7060, Balu761, and Dow2A120 were evaluated in four places, using a randomized block experimental design, with three replicates. The hybrids $\mathrm{LGS}_{3} \times \mathrm{LGS}_{9}, \mathrm{LGS}_{2} \mathrm{xLGS}_{6}, \mathrm{LGS}_{2} \times \mathrm{xGS}_{4}$ and $\mathrm{LGS}_{2} \mathrm{xLGS}$ showed an excellent performance in comparison with the controls, for the different characters evaluated. The lines with higher frequency of favorable alleles were $\mathrm{LGS}_{2}, \mathrm{LGS}_{9}, \mathrm{LGS}_{4}$ and $\mathrm{LGS}_{3}$. The additive genetic effects are the most important for the resistance to $P$. maydis and for ear height, while nonadditive genetic effects are more important for yield, plant height, resistance to E. turcicum, P. zeae and P. polysora.
\end{abstract}

Index terms: Exserohilum turcicum, Phaeosphaeria maydis, Physopella zeae, Puccinia polysora, Zea mays, genetic effect.

\section{Introdução}

As doenças foliares do milho (Zea mays L.), ferrugem-polissora (Puccinia polysora Underw.), ferrugem tropical [Physopella zeae (Mains) Cummins \& Ramachar], mancha-branca [Phaeosphaeria maydis (P. Henn.) Rane, Payak \& Renfro] e helmintosporiose [Exserohilum turcicum (Pass.) Leonard \& Suggs], cresceram em importância no Brasil a partir de 1990, pelo aumento da frequência, severidade e danos qualitativos e quantitativos à produção da cultura. As ações de melhoramento genético focadas apenas na produtividade e precocidade, sem considerar a resistência genética a doenças foliares; o uso de híbridos e variedades suscetíveis em sistemas de plantio direto; a sucessão de cultivos de primeira e segunda safra; a manutenção da umidade por irrigações; e o aumento da área cultivada, inclusive em áreas de risco de epidemias, foram fatores decisivos para esse resultado (Pinto, 2004; Emygdio et al., 2007).

Os programas de melhoramento genético de milho têm com um de seus objetivos o desenvolvimento de linhagens resistentes a doenças foliares (Silveira et al., 2006), uma vez que esta tem sido o meio mais 
eficaz de controle das doenças do milho (Von Pinho et al., 2001). Diferentes estudos mostram a existência de variabilidade genética quanto à resistência à mancha-branca, e que a ação gênica aditiva é mais importante do que os efeitos não aditivos (Paterniani et al., 2000; Carson, 2001; Pegoraro et al., 2002; Silva \& Moro, 2004; Derera et al., 2007; Lopes et al., 2007; Guimarães et al., 2009; Vivek et al., 2010). A ação gênica aditiva também se mostrou mais importante na maioria dos estudos de herança da resistência à helmintosporiose (Carson, 1995; Vivek et al., 2010) e à ferrugem causada por $P$. zeae e $P$. polysora (Paterniani et al., 2000; Silva et al., 2001; Vivek et al., 2010).

As estimativas de capacidade de combinação, obtidas por meio de esquemas de cruzamentos dialélicos, são importantes na escolha de genitores e no entendimento dos efeitos genéticos envolvidos na determinação dos caracteres (Cruz et al., 2004). Além disso, são também empregadas em estudos de resistência genética a doenças e na identificação de fontes de resistência (Carson, 2001; Pegoraro et al., 2002; Silva et al., 2003; Silva \& Moro, 2004).

O objetivo deste trabalho foi identificar o tipo de ação gênica predominante quanto à resistência a $P$. maydis, E. turcicum, $P$. zeae e $P$. polysora, e determinar o potencial genético de linhagens endogâmicas de milho (Z. mays) na obtenção de híbridos com elevado desempenho agronômico e resistência a doenças foliares.

\section{Material e Métodos}

Foram realizados cruzamentos dialélicos entre dez linhagens endogâmicas - $\mathrm{LGS}_{1}$ a $\mathrm{LGS}_{10}$ - do Programa de Melhoramento Genético da Empresa GS Pesquisas (Apucarana, PR), na Fazenda Alto Alegre, Pitangueiras, PR (a $23^{\circ} 13^{\prime} 50^{\prime \prime} \mathrm{S}, 51^{\circ} 35^{\prime} 08^{\prime \prime} \mathrm{W}$ e altitude de $660 \mathrm{~m}$ ), durante a safra 2008/2009. As linhagens foram previamente selecionadas, em relação ao seu desempenho per se, quanto à resistência a doenças foliares e demais características agronômicas.

Foram obtidos 41 híbridos experimentais, que foram avaliados em quatro locais, tendo-se utilizado o delineamento de blocos ao acaso com três repetições, em parcelas de fileiras simples de $5 \mathrm{~m}$ de comprimento, no espaçamento de $0,80 \times 0,20 \mathrm{~m}$, conforme as recomendações técnicas de controle fitossanitário e de produção de híbridos simples. Como testemunhas, foram empregados híbridos comerciais com diferentes padrões de resistência: P3069, suscetível a $P$. maydis, $P$. zeae e $P$. polysora e medianamente resistente a E. turcicum; P30F90, suscetível a E. turcicum e $P$. zeae, medianamente suscetível a $P$. maydis e medianamente resistente a $P$. polysora; BG 7060 , suscetível a $P$. polysora, medianamente suscetível a $P$. maydis e medianamente resistente a E. turcicum e $P$. zeae; Balu761, medianamente suscetível a $P$. maydis, medianamente resistente a $E$. turcicum e sem informações sobre resistência a $P$. zeae e $P$. polysora; e Dow2A120, suscetível a $P$. maydis, medianamente resistente a $E$. turcicum e $P$. polysora e sem informações quanto à resistência a $P$. zeae.

As datas e locais de instalação dos experimentos tiveram por finalidade favorecer a ocorrência de epidemias naturais de doenças foliares. Foram efetuadas semeaduras em: 21 de novembro de 2009, em Pitangueiras, PR (a $23^{\circ} 13^{\prime} 50^{\prime \prime} \mathrm{S}$ e $51^{\circ} 35^{\prime} 08^{\prime \prime} \mathrm{W}$ e à altitude de $660 \mathrm{~m}$ ); 29 de janeiro de 2010, em Sorriso,

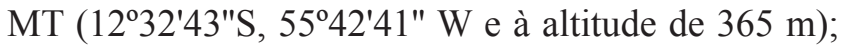
05 de fevereiro de 2010, em Jaciara, MT (a 15 $57^{\prime} 55^{\prime \prime}$ ', $54^{\circ} 58^{\prime} 06^{\prime \prime} \mathrm{W}$ e à altitude de $\left.367 \mathrm{~m}\right) ; 24$ de fevereiro de 2010, em Dourados, MS (a 22 ${ }^{\circ} 13^{\prime} 16^{\prime \prime} \mathrm{S}, 54^{\circ} 48^{\prime} 20^{\prime \prime}$ $\mathrm{W}$ e à altitude de $430 \mathrm{~m}$ ). Contudo, epidemias de mancha-branca, helmintosporiose, e ferrugens tropical e polissora foram observadas somente em Pitangueiras, PR, e da mancha-branca em Sorriso, MT, onde foram realizadas as avaliações de severidade das doenças foliares.

Os caracteres avaliados foram: altura da planta $(\mathrm{cm})$, tomada da superfície do solo até a inserção da folha bandeira; altura da espiga $(\mathrm{cm})$, tomada da superfície do solo até a inserção da espiga principal; produtividade de grãos $\left(\mathrm{Mg} \mathrm{ha}^{-1}\right)$, corrigida para umidade de 14\%; e densidade populacional ideal de 62.500 plantas ha ${ }^{-1}$, segundo a metodologia de correção de estande ideal por covariâncias. Para avaliação da severidade das doenças foliares, foram atribuídas notas de severidade para as doenças mancha-branca, helmintosporiose, ferrugem tropical e ferrugem-polissora, com o auxílio do Guia Agroceres de Sanidade (1996), em escala de 1 (altamente resistente) a 9 (altamente suscetível).

As análises individuais de variâncias por local, com base na média de tratamentos, foram realizadas com decomposição dos efeitos de tratamentos em efeitos de testemunhas ( $\mathrm{T}$ ), híbridos experimentais (H) e no contraste T vs. H. Os graus de liberdade de 
híbridos experimentais foram desdobrados por meio da análise dialélica, segundo o método IV proposto por Griffing (1956), de acordo com o modelo: $\mathrm{Y}_{\mathrm{ii}}=\mathrm{m}+\mathrm{g}_{\mathrm{i}}+\mathrm{g}_{\mathrm{i}^{\prime}}+\mathrm{s}_{\mathrm{ii}}+\overline{\mathrm{e}}_{\mathrm{ii}}$, em que: $\mathrm{Y}_{\mathrm{ii}}$ é o valor médio da combinação híbrida da linhagem $\mathrm{LGS}_{\mathrm{i}}$ com a LGS $;$; $\mathrm{m}$ é a média geral das combinações híbridas; $g_{i}$ e $g_{i^{\prime}}$ são os efeitos da capacidade geral de combinação (CGC) da i-ésima linhagem $L G S_{i}$ e i'-ésima linhagem $L G S_{i}$, respectivamente; $\mathrm{s}_{\mathrm{ii}}$ é o efeito da capacidade específica de combinação (CEC) para os cruzamentos entre os genitores de ordem i e i'; e $\overline{\mathrm{e}}_{\mathrm{i}}$ ' é o erro experimental médio.

A análise de variância do dialelo e as estimativas de $g_{i}, g_{i}$, e $s_{i i}$, foram realizadas por meio de álgebra de matrizes, pelo modelo matricial $\mathrm{Y}=\mathrm{X} \beta+\varepsilon$, em que: Y é o vetor dos dados de médias observadas para os híbridos experimentais; $\mathrm{X}$ é a matriz de incidência com valores de 0 e 1 , relacionados aos parâmetros $\mathrm{m}, \mathrm{g}_{\mathrm{i}}, \mathrm{g}_{\mathrm{i}}$, e $\mathrm{s}_{\mathrm{ii}}$; $\beta$ é vetor dos parâmetros $\mathrm{m}, \mathrm{g}_{\mathrm{i}}, \mathrm{g}_{\mathrm{i}}$, e $\mathrm{s}_{\mathrm{ii}}$; , e $\varepsilon$ é o vetor que representa o erro associado às médias $\left(\overline{\mathrm{e}}_{\mathrm{ii}}\right)$.

$\mathrm{O}$ desvio-padrão (DP) das estimativas de CGC $\left[D P\left(g_{i}\right)\right]$ e de seus contrastes $\left[D P\left(g_{i}-g_{i}\right)\right]$ foi estimado a partir dos quadrados médios do erro $\left(\mathrm{QM}_{\text {Erro }}\right)$, obtidos para os dados não transformados, e do número $\mathrm{n}$ de linhagens envolvidas no dialelo, por meio das seguintes expressões: $\mathrm{DP}\left(\mathrm{g}_{\mathrm{i}}\right)=\left\{(\mathrm{n}-1) \mathrm{QM}_{\mathrm{Erro}} /[\mathrm{n}(\mathrm{n}-2)]\right\}^{0,5}$ e DP $\left(g_{\mathrm{i}}-\mathrm{g}_{\mathrm{i}}\right)=\left[2 \mathrm{QM}_{\text {Erro }} /(\mathrm{n}-2)\right]^{0,5}$.

De modo semelhante, os desvios-padrão das estimativas de CEC $\left[\mathrm{DP}\left(\mathrm{s}_{\mathrm{iii}}\right)\right]$, dos contrastes de estimativas de CEC entre híbridos obtidos do cruzamento entre um genitor $i$, comum aos diferentes genitores i' e $\mathrm{k}\left[\mathrm{DP}\left(\mathrm{s}_{\mathrm{ii}},-\mathrm{s}_{\mathrm{ik}}\right)\right]$, e dos contrastes de estimativas de CEC de híbridos simples, sem a presença de genitores (i, i', k e k') em comum $\left[\mathrm{DP}\left(\mathrm{s}_{\mathrm{ii}},-\mathrm{s}_{\mathrm{kk}}\right)\right]$, foram estimados por meio das expressões $\mathrm{DP}\left(\mathrm{s}_{\mathrm{ii}}\right)=\left[(\mathrm{n}-3) \mathrm{QM}_{\mathrm{Erro}} /(\mathrm{n}-1)\right]^{0,5}, \mathrm{DP}\left(\mathrm{s}_{\mathrm{ii}},-\mathrm{s}_{\mathrm{ik}}\right)=$ $\left[2(\mathrm{n}-3) \mathrm{QM}_{\mathrm{Erro}} /(\mathrm{n}-2)\right]^{0,5}$ e DP $\left(\mathrm{s}_{\mathrm{ii}},-\mathrm{s}_{\mathrm{kk}}\right)=[2(\mathrm{n}-4)$ $\left.\mathrm{QM}_{\text {Erro }} /(\mathrm{n}-2)\right]^{0,5}$.

As análises de variâncias das notas de severidade foram realizadas com dados transformados para $(\mathrm{x})^{0,5} \mathrm{e}$ as médias e estimativas de parâmetros do dialelo foram apresentadas com base nos dados não transformados.

As análises de variâncias conjuntas dos locais e os respectivos desdobramentos dos cruzamentos e das interações cruzamentos $\mathrm{x}$ locais foram realizados conforme Miranda Filho \& Vencovsky (1995), tendo-se considerado os efeitos fixos de genótipos e ambientes. $\mathrm{O}$ agrupamento de médias dos tratamentos, dentro de cada local, foi realizado pelo teste de Scott \& Knott, a $5 \%$ de probabilidade.

As estimativas dos componentes quadráticos que expressam a variabilidade genética dos genótipos estudados, em termos de capacidade geral de combinação e de capacidade específica de combinação, foram obtidas a partir das expressões $\phi_{\mathrm{g}}=\left(\mathrm{QM}_{\mathrm{CGC}}-\mathrm{QM}_{\mathrm{Erro}}\right) /$ $(n-2)$ e $\phi_{\mathrm{s}}=\left(\mathrm{QM}_{\mathrm{CEC}}-\mathrm{QM}_{\mathrm{Erro}}\right)$, em que: $\mathrm{QM}_{\mathrm{CGC}}$ é o quadrado médio da capacidade geral de combinação; $\mathrm{QM}_{\mathrm{CEC}}$ é o quadrado médio da capacidade específica de combinação; e n é o número de linhagens envolvidas no dialelo.

As análises de variâncias dos experimentos individuais foram realizadas por meio do programa SAS (SAS Institute, 2002) e o teste de Scott \& Knott com o uso do programa Genes (Cruz, 2001).

\section{Resultados e Discussão}

Houve efeito significativo de local quanto à produtividade, altura de plantas e de espigas (Tabela 1), com as maiores médias em Pitangueiras, onde o experimento foi instalado durante a safra (Tabela 2). Normalmente, na safrinha, o potencial de rendimento de grãos é mais baixo, em consequência da menor quantidade de radiação solar durante o período de enchimento de grãos e da maior incidência de doenças foliares e de colmo (Forsthofer et al., 2006). Contudo, não foram observadas, no presente estudo, diferenças significativas entre as médias de notas de severidade da mancha-branca em Pitangueiras e Sorriso (Tabela 1). Em Pitangueiras, ocorreram epidemias de mancha-branca, helmintosporiose, e de ferrugens tropical e polissora, e, em Sorriso, somente houve epidemia de mancha-branca. Isto pode ter ocorrido em razão do cultivo contínuo de milho na Estação Experimental da Empresa GS Sementes, o que teria favorecido a manutenção das populações de patógenos na área.

Os efeitos dos tratamentos foram significativos em todos os caracteres avaliados (Tabela 1). No desdobramento, foi observada ausência de significância, entre as testemunhas, apenas para altura de planta. Para o contraste entre testemunhas e híbridos experimentais ( $\mathrm{T} \times \mathrm{H})$, houve significância apenas para notas de severidade de ferrugens (polissora e tropical) e helmintosporiose, em que as testemunhas foram ligeiramente mais suscetíveis às ferrugens do que os 
híbridos experimentais, e mais resistentes a E. turcicum. A não detecção de efeitos significativos dos contrastes de produtividade, altura de planta e notas de severidade à mancha-branca, inclusive para interação com local $[\mathrm{L} \times(\mathrm{T} \times \mathrm{H})]$, mostram um desempenho médio semelhante entre os híbridos do dialelo e as testemunhas para estas características (Tabelas 1 e 2), o que indica que os híbridos experimentais atingem as exigências de padrões agronômicos para exploração comercial.

Houve efeitos significativos de híbridos e da interação híbrido x local para todas as características, o que indica um comportamento diferenciado dos genótipos em cada local (Tabela 1). Embora tenha havido interações significativas, comparando-se os híbridos experimentais com as melhores médias entre as testemunhas, quanto aos diferentes caracteres avaliados nos locais, os híbridos resultantes dos cruzamentos $\mathrm{LGS}_{3} \mathrm{xLGS}, \mathrm{LGS}_{2} \times \mathrm{LGS}_{6}, \mathrm{LGS}_{2} \mathrm{xLGS}$ e $\mathrm{LGS}_{2} \mathrm{XLGS}_{3}$ tiveram os melhores desempenhos em produtividade (Tabela 2), com destaque para $\mathrm{LGS}_{3} \mathrm{xLGS}_{9}$, com um dos melhores níveis de resistência às doenças estudadas. $\mathrm{O}$ híbrido $\mathrm{LGS}_{2} \mathrm{XLGS}_{4}$ teve desempenho superior também quanto à produtividade, em todos os locais, além de ter se mostrado um dos genótipos mais resistentes à mancha-branca. Apenas em Sorriso o híbrido $\mathrm{LGS}_{2} \mathrm{xLGS}_{3}$ não apresentou produtividade superior, mas mostrou-se resistente à ferrugem-polissora, com plantas mais baixas e com menor altura de espiga. Interações significativas de genótipos $\mathrm{x}$ ambientes, quanto à resistência a doenças causadas por $P$. maydis, também foram observadas por Paterniani et al. (2000), Carson (2001), Lopes et al. (2007), Guimarães et al. (2009) e Vivek et al. (2010).

Foram observados efeitos significativos de capacidade geral de combinação (CGC), e de suas interações com locais, em todas as características avaliadas (Tabela 1). Os efeitos significativos de CGC indicam um comportamento médio diferente de cada genitor,

Tabela 1. Quadrados médios com base em médias de tratamentos, níveis de significância e componentes quadráticos das capacidades geral $\left(\phi_{\mathrm{g}}\right)$ e específica $\left(\phi_{\mathrm{s}}\right)$ de combinação, para as características produtividade de grãos $(\mathrm{PG}), \mathrm{Mg} \mathrm{ha}^{-1}$, altura de planta (AP) e altura de espiga (AE) em cm, avaliadas em quatro locais (Pitangueiras, Sorriso, Jaciara e Dourados), e notas de severidade de mancha-branca (MB), em dois locais (Pitangueiras e Sorriso), e de helmintosporiose (ET), ferrugem tropical (PHY) e ferrugem-polissora (FP) em Pitangueiras ${ }^{(1)}$.

\begin{tabular}{|c|c|c|c|c|c|c|c|c|c|c|}
\hline \multirow[t]{2}{*}{ Fonte de variação } & \multicolumn{4}{|c|}{ Análise de quatro locais } & \multicolumn{2}{|c|}{ Análise de dois locais } & \multicolumn{4}{|c|}{ Análise individual em Pitangueiras ${ }^{(2)}$} \\
\hline & GL & PG & AP & $\mathrm{AE}$ & GL & $\mathrm{MB}^{(2)}$ & GL & ET & PHY & FP \\
\hline Local (L) & 3 & $103,48 * *$ & $501,86^{* *}$ & $323,61 * *$ & 1 & $7813,8^{\text {ns }}$ & - & - & - & - \\
\hline Tratamento & 45 & $1,0436^{* *}$ & $113,57 * *$ & $144,72 * *$ & 45 & $885,71 * *$ & 45 & $259,63 * *$ & $514,16^{* *}$ & $672,23 * *$ \\
\hline Testemunha $(\mathrm{T})$ & 4 & $2,9099 * *$ & $65,213^{\text {ns }}$ & $249,41 * *$ & 4 & $1254,3^{* *}$ & 4 & $532,67^{* *}$ & $1101,2 * *$ & $1169,8^{* *}$ \\
\hline $\mathrm{T} \times \mathrm{H}$ & 1 & $0,3995^{\text {ns }}$ & $50,111^{\mathrm{ns}}$ & $108,06^{\mathrm{ns}}$ & 1 & $117,23^{\text {ns }}$ & 1 & $730,70^{* *}$ & $3954,8 * *$ & $1340,6^{* *}$ \\
\hline Híbridos $(\mathrm{H})$ & 40 & $0,8731^{* *}$ & $120,00^{* *}$ & $135,17 * *$ & 40 & $868,07 * *$ & 40 & $220,55^{* *}$ & $369,44 * *$ & $605,77 * *$ \\
\hline CGC & 9 & $1,8475^{* *}$ & $275,25^{* *}$ & $362,54 * *$ & 9 & $2741,9 * *$ & 9 & $416,11 * *$ & $770,06^{* *}$ & $1330,7^{* *}$ \\
\hline CEC & 31 & $0,5902 * *$ & $74,923 * *$ & $69,157 * *$ & 31 & $324,05^{* *}$ & 31 & $163,77 * *$ & $253,13 * *$ & $395,31 * *$ \\
\hline $\mathrm{L} x$ tratamentos & 135 & $0,6987 * *$ & $69,218 * *$ & $74,530 * *$ & 45 & $515,73 * *$ & - & - & - & - \\
\hline $\mathrm{L} \times \mathrm{T}$ & 12 & $1,2994 * *$ & $73,002 * *$ & $83,261 * *$ & 4 & $1220,4^{* *}$ & - & - & - & - \\
\hline $\mathrm{L} \times(\mathrm{T} \times \mathrm{H})$ & 3 & $0,4103^{\text {ns }}$ & $70,288^{\text {ns }}$ & $144,87^{* *}$ & 1 & $31,053^{\text {ns }}$ & - & - & - & - \\
\hline L x híbrido $(\mathrm{H})$ & 120 & $0,6459^{* *}$ & $68,812 * *$ & $71,899 * *$ & 40 & $457,38^{* *}$ & - & - & - & - \\
\hline L x CGC & 27 & $1,2236^{* *}$ & $118,38 * *$ & $126,48^{* *}$ & 9 & $1253,5^{* *}$ & - & - & - & - \\
\hline L x CEC & 93 & $0,4781 * *$ & $54,422 * *$ & $56,052 * *$ & 31 & $226,25^{* *}$ & - & - & - & - \\
\hline Erro & 326 & 0,2736 & 31,711 & 32,336 & 176 & 80,516 & 86 & 61,468 & 94,438 & 155,54 \\
\hline \multirow[t]{2}{*}{ CV (\%) } & & 13,8 & 4,9 & 8,5 & & 7,9 & & 6,3 & 7,7 & 10,9 \\
\hline & \multicolumn{10}{|c|}{ Componentes quadráticos } \\
\hline$\phi_{\mathrm{g}}$ & & 0,1967 & 30,442 & 41,275 & & 332,67 & & 44,330 & 84,452 & 146,89 \\
\hline$\phi_{\mathrm{s}}$ & & 0,3166 & 43,212 & 36,821 & & 243,53 & & 102,30 & 158,69 & 239,77 \\
\hline$\phi_{\mathrm{g}} / \phi_{\mathrm{s}}$ & & 0,6214 & 0,7044 & 1,1209 & & 1,3660 & & 0,4333 & 0,5321 & 0,6126 \\
\hline
\end{tabular}

(1)ns Não significativo. ** e *Significativo a 1 e $5 \%$ de probabilidade, respectivamente. ${ }^{(2)}$ Os quadrados médios de notas de severidade a doenças foram transformados para $(\mathrm{x})^{0,5}$, multiplicados por $10^{-4}$. CGC, capacidade geral de combinação; CEC, capacidade específica de combinação; $\phi_{\mathrm{g}}$, componentes quadráticos que expressam a variabilidade genética em termos de capacidade geral de combinação; $\phi_{\mathrm{s}}$, componentes quadráticos que expressam a variabilidade genética em termos de capacidade específica de combinação. 
Tabela 2. Produtividade de grãos $\left(\mathrm{Mg} \mathrm{ha}^{-1}\right)$, altura de planta $(\mathrm{cm})$, altura de espiga $(\mathrm{cm})$, e notas de severidade de mancha-branca (MB), helmintosporiose (ET), ferrugem tropical (PHY) e de ferrugem-polissora (FP), de 41 híbridos avaliados em Pitangueiras (PIT), Sorriso (SO), Jaciara (JA) e Dourados (DO) ${ }^{(1)}$.

\begin{tabular}{|c|c|c|c|c|c|c|c|c|c|c|c|c|c|c|c|c|c|}
\hline \multirow[t]{2}{*}{ Híbrido } & \multicolumn{4}{|c|}{ Produtividade } & \multicolumn{4}{|c|}{ Altura da planta } & \multicolumn{4}{|c|}{ Altura da espiga } & \multicolumn{2}{|c|}{ MB } & \multirow{2}{*}{$\begin{array}{l}\text { ET } \\
\text { PIT }\end{array}$} & \multirow{2}{*}{$\begin{array}{l}\text { PHY } \\
\text { PIT }\end{array}$} & \multirow{2}{*}{$\begin{array}{l}\text { FP } \\
\text { PIT }\end{array}$} \\
\hline & PIT & SO & JA & DO & PIT & SO & JA & DO & PIT & $\mathrm{SO}$ & JA & DO & PIT & SO & & & \\
\hline $\mathrm{LGS}_{1} \times \mathrm{LGS}_{2}$ & $9,71 b$ & $5,58 \mathrm{a}$ & $6,10 \mathrm{a}$ & $4,53 \mathrm{a}$ & $265 \mathrm{a}$ & $188 \mathrm{a}$ & $192 a$ & $171 \mathrm{c}$ & $153 \mathrm{a}$ & $77 \mathrm{a}$ & $108 \mathrm{a}$ & $88 \mathrm{~b}$ & $3,5 \mathrm{e}$ & $4,2 \mathrm{a}$ & $4,5 b$ & $3,3 \mathrm{e}$ & $3,0 \mathrm{~b}$ \\
\hline $\mathrm{LGS}_{1} \times \mathrm{LGS}_{3}$ & $8,36 \mathrm{c}$ & $4,88 \mathrm{~b}$ & $4,74 \mathrm{a}$ & $5,86 \mathrm{a}$ & $248 \mathrm{a}$ & $173 a$ & $183 a$ & $173 b$ & $143 \mathrm{a}$ & $73 b$ & $92 \mathrm{a}$ & $88 \mathrm{~b}$ & $7,0 \mathrm{~b}$ & $5,0 \mathrm{a}$ & $4,7 \mathrm{~b}$ & $3,5 \mathrm{e}$ & $2,5 \mathrm{~b}$ \\
\hline $\mathrm{LGS}_{1} \times \mathrm{LGS}_{4}$ & $10,25 b$ & $4,51 \mathrm{~b}$ & $5,13 \mathrm{a}$ & $5,64 \mathrm{a}$ & $250 \mathrm{a}$ & $165 \mathrm{~b}$ & $188 \mathrm{a}$ & $170 \mathrm{c}$ & $148 \mathrm{a}$ & $67 b$ & $93 a$ & $97 \mathrm{a}$ & $5,7 \mathrm{c}$ & $3,5 \mathrm{a}$ & $3,8 \mathrm{c}$ & $3,0 \mathrm{e}$ & $3,5 \mathrm{~b}$ \\
\hline $\mathrm{LGS}_{1} \times \mathrm{LGS}_{5}$ & $9,37 \mathrm{~b}$ & $6,03 a$ & $5,91 \mathrm{a}$ & $5,68 \mathrm{a}$ & $243 a$ & $168 b$ & $192 \mathrm{a}$ & $182 \mathrm{a}$ & $135 b$ & $65 \mathrm{~b}$ & $98 \mathrm{a}$ & $102 a$ & $4,0 \mathrm{e}$ & $3,5 \mathrm{a}$ & $5,0 \mathrm{~b}$ & $5,0 \mathrm{c}$ & $3,5 b$ \\
\hline $\mathrm{LGS}_{1} \mathrm{xLGS}_{6}$ & $7,50 \mathrm{c}$ & $6,14 \mathrm{a}$ & $5,32 \mathrm{a}$ & $5,81 \mathrm{a}$ & $227 b$ & $175 \mathrm{a}$ & $193 a$ & $168 \mathrm{c}$ & $133 b$ & $80 \mathrm{a}$ & $93 a$ & $90 \mathrm{~b}$ & $5,0 \mathrm{~d}$ & $4,7 \mathrm{a}$ & $5,0 \mathrm{~b}$ & $3,5 \mathrm{e}$ & $3,3 b$ \\
\hline $\mathrm{LGS}_{1} \times \mathrm{LGS}_{7}$ & $7,70 \mathrm{c}$ & $5,55 \mathrm{a}$ & $6,60 \mathrm{a}$ & $5,80 \mathrm{a}$ & $247 \mathrm{a}$ & $183 a$ & $192 \mathrm{a}$ & $163 d$ & $142 \mathrm{a}$ & $80 \mathrm{a}$ & $92 \mathrm{a}$ & $75 \mathrm{c}$ & $6,0 \mathrm{c}$ & $4,0 \mathrm{a}$ & $5,0 \mathrm{~b}$ & $4,7 \mathrm{~d}$ & $2,3 \mathrm{~b}$ \\
\hline $\mathrm{LGS}_{1} \mathrm{xLGS}_{9}$ & $7,54 \mathrm{c}$ & $6,68 \mathrm{a}$ & $6,31 \mathrm{a}$ & $5,56 \mathrm{a}$ & $238 b$ & $167 \mathrm{~b}$ & $190 \mathrm{a}$ & $163 d$ & $138 \mathrm{~b}$ & $77 \mathrm{a}$ & $102 \mathrm{a}$ & $63 d$ & $3,7 \mathrm{e}$ & $4,5 \mathrm{a}$ & $4,5 b$ & $4,7 d$ & $3,5 \mathrm{~b}$ \\
\hline $\mathrm{LGS}_{1} \times \mathrm{LGS}_{10}$ & $9,42 b$ & $5,27 \mathrm{~b}$ & $6,96 \mathrm{a}$ & $5,63 \mathrm{a}$ & $250 \mathrm{a}$ & $170 \mathrm{~b}$ & $185 \mathrm{a}$ & $166 \mathrm{c}$ & $132 \mathrm{~b}$ & $73 b$ & $95 \mathrm{a}$ & $78 \mathrm{c}$ & $5,0 \mathrm{~d}$ & $3,5 \mathrm{a}$ & $5,5 \mathrm{a}$ & $4,5 \mathrm{~d}$ & $4,0 \mathrm{a}$ \\
\hline $\mathrm{LGS}_{2} \times \mathrm{XGS}_{3}$ & $10,39 a$ & $5,08 \mathrm{~b}$ & $6,50 \mathrm{a}$ & $5,20 \mathrm{a}$ & $237 b$ & $162 b$ & $193 a$ & $182 a$ & $123 \mathrm{c}$ & $72 b$ & $98 \mathrm{a}$ & $95 \mathrm{~b}$ & $4,5 \mathrm{~d}$ & $3,2 b$ & $4,7 \mathrm{~b}$ & $5,0 \mathrm{c}$ & $3,5 \mathrm{~b}$ \\
\hline $\mathrm{LGS}_{2} \times \mathrm{LGS}_{4}$ & $10,64 \mathrm{a}$ & $5,73 a$ & $5,22 \mathrm{a}$ & $6,67 \mathrm{a}$ & $253 a$ & $183 a$ & $183 a$ & $163 d$ & $153 a$ & $83 a$ & $92 \mathrm{a}$ & $95 b$ & $2,5 \mathrm{f}$ & $2,8 \mathrm{~b}$ & $4,5 b$ & $5,0 \mathrm{c}$ & $4,3 \mathrm{a}$ \\
\hline $\mathrm{LGS}_{2} \times \mathrm{LGS}_{5}$ & $8,58 \mathrm{c}$ & $5,46 a$ & $6,62 \mathrm{a}$ & $5,76 \mathrm{a}$ & $255 \mathrm{a}$ & $162 b$ & $183 a$ & $158 \mathrm{~d}$ & $148 \mathrm{a}$ & $70 \mathrm{~b}$ & $95 a$ & $75 \mathrm{c}$ & $3,0 \mathrm{f}$ & $3,0 \mathrm{~b}$ & $4,5 b$ & $5,0 \mathrm{c}$ & $3,5 \mathrm{~b}$ \\
\hline $\mathrm{LGS}_{2} \times \mathrm{LGS}_{6}$ & $11,58 \mathrm{a}$ & $6,59 a$ & $6,35 \mathrm{a}$ & $5,62 \mathrm{a}$ & $260 \mathrm{a}$ & $187 \mathrm{a}$ & $198 \mathrm{a}$ & $163 d$ & $163 a$ & $87 a$ & $102 \mathrm{a}$ & $83 \mathrm{c}$ & $4,0 \mathrm{e}$ & $3,2 b$ & $5,0 \mathrm{~b}$ & $4,5 \mathrm{~d}$ & $5,7 \mathrm{a}$ \\
\hline $\mathrm{LGS}_{2} \times \mathrm{LGS}_{7}$ & $9,46 \mathrm{~b}$ & $5,52 \mathrm{a}$ & $5,38 \mathrm{a}$ & $7,25 \mathrm{a}$ & $268 \mathrm{a}$ & $190 \mathrm{a}$ & $193 a$ & $189 a$ & $162 \mathrm{a}$ & $98 \mathrm{a}$ & $95 \mathrm{a}$ & $91 b$ & $5,0 \mathrm{~d}$ & $3,0 \mathrm{~b}$ & $5,0 \mathrm{~b}$ & $4,5 \mathrm{~d}$ & $5,2 \mathrm{a}$ \\
\hline $\mathrm{LGS}_{2} \mathrm{XLGS}_{9}$ & $9,72 b$ & $6,20 \mathrm{a}$ & $5,29 a$ & $4,86 a$ & $247 \mathrm{a}$ & $172 b$ & $183 a$ & $171 \mathrm{c}$ & $137 \mathrm{~b}$ & $80 a$ & $83 a$ & $95 \mathrm{~b}$ & $3,0 \mathrm{f}$ & $3,5 \mathrm{a}$ & $4,5 \mathrm{~b}$ & $4,5 \mathrm{~d}$ & $3,2 \mathrm{~b}$ \\
\hline $\mathrm{LGS}_{2} \mathrm{xLGS}_{10}$ & $9,07 \mathrm{~b}$ & $5,58 \mathrm{a}$ & $5,56 \mathrm{a}$ & $5,75 \mathrm{a}$ & $250 \mathrm{a}$ & $178 \mathrm{a}$ & $187 \mathrm{a}$ & $168 \mathrm{c}$ & $158 \mathrm{a}$ & $80 a$ & $97 a$ & $92 \mathrm{~b}$ & $3,0 \mathrm{f}$ & $3,7 \mathrm{a}$ & $4,7 \mathrm{~b}$ & $4,7 d$ & $4,5 \mathrm{a}$ \\
\hline $\mathrm{LGS}_{3} \times \mathrm{xGS}_{4}$ & $9,14 b$ & $5,21 \mathrm{~b}$ & $5,96 \mathrm{a}$ & $5,49 a$ & $225 b$ & $162 b$ & $193 a$ & $166 \mathrm{c}$ & $123 \mathrm{c}$ & $70 \mathrm{~b}$ & $92 a$ & $83 c$ & $4,0 \mathrm{e}$ & $3,7 \mathrm{a}$ & $3,3 \mathrm{~d}$ & $3,8 \mathrm{~d}$ & $2,7 \mathrm{~b}$ \\
\hline $\mathrm{LGS}_{3} \times \mathrm{LGS}_{5}$ & $9,29 b$ & $5,33 b$ & $4,26 \mathrm{a}$ & $6,35 \mathrm{a}$ & $222 b$ & $163 \mathrm{~b}$ & $188 \mathrm{a}$ & $166 \mathrm{c}$ & $110 \mathrm{c}$ & $63 b$ & $92 a$ & $78 \mathrm{c}$ & $4,3 \mathrm{e}$ & $2,8 \mathrm{~b}$ & $3,5 \mathrm{~d}$ & $4,0 \mathrm{~d}$ & $3,0 \mathrm{~b}$ \\
\hline $\mathrm{LGS}_{3} \times \mathrm{LGS}_{6}$ & $8,97 \mathrm{~b}$ & $5,00 \mathrm{~b}$ & $5,63 \mathrm{a}$ & $5,71 \mathrm{a}$ & $223 b$ & $167 \mathrm{~b}$ & $192 a$ & $173 b$ & $123 \mathrm{c}$ & $67 \mathrm{~b}$ & $97 a$ & $78 \mathrm{c}$ & $5,3 \mathrm{c}$ & $3,5 \mathrm{a}$ & $5,7 \mathrm{a}$ & $4,0 \mathrm{~d}$ & $3,0 \mathrm{~b}$ \\
\hline $\mathrm{LGS}_{3} \times \mathrm{LGS}_{7}$ & $7,54 \mathrm{c}$ & $5,05 \mathrm{~b}$ & $6,34 a$ & $6,32 \mathrm{a}$ & $233 b$ & $170 \mathrm{~b}$ & $198 \mathrm{a}$ & $168 \mathrm{c}$ & $132 \mathrm{~b}$ & $75 a$ & $100 \mathrm{a}$ & $98 \mathrm{a}$ & $8,0 \mathrm{a}$ & $3,5 \mathrm{a}$ & $4,5 b$ & $3,0 \mathrm{e}$ & $3,0 \mathrm{~b}$ \\
\hline $\mathrm{LGS}_{3} \mathrm{xLGS}_{9}$ & $11,36 \mathrm{a}$ & $5,81 \mathrm{a}$ & $6,29 a$ & $5,84 \mathrm{a}$ & $235 b$ & $167 \mathrm{~b}$ & $188 \mathrm{a}$ & $183 a$ & $130 \mathrm{~b}$ & $65 b$ & $93 a$ & $82 \mathrm{c}$ & $4,5 \mathrm{~d}$ & $3,2 b$ & $4,5 b$ & $3,5 \mathrm{e}$ & $3,0 \mathrm{~b}$ \\
\hline $\mathrm{LGS}_{3} \times \mathrm{LGS}_{10}$ & $8,29 \mathrm{c}$ & $5,00 \mathrm{~b}$ & $5,32 \mathrm{a}$ & $5,58 \mathrm{a}$ & $233 b$ & $162 b$ & $193 a$ & $167 \mathrm{c}$ & $128 \mathrm{~b}$ & $55 \mathrm{~b}$ & $98 \mathrm{a}$ & $78 \mathrm{c}$ & $5,3 \mathrm{c}$ & $3,0 \mathrm{~b}$ & $5,0 \mathrm{~b}$ & $4,7 \mathrm{~d}$ & $2,3 b$ \\
\hline $\mathrm{LGS}_{4} \times \mathrm{LGS}_{5}$ & $8,67 \mathrm{c}$ & $5,54 \mathrm{a}$ & $4,81 \mathrm{a}$ & $6,16 a$ & $228 b$ & $165 \mathrm{~b}$ & $172 \mathrm{a}$ & $181 \mathrm{a}$ & $127 \mathrm{~b}$ & $68 b$ & $100 \mathrm{a}$ & $110 \mathrm{a}$ & $3,0 \mathrm{f}$ & $3,5 \mathrm{a}$ & $3,0 \mathrm{~d}$ & $4,0 \mathrm{~d}$ & $3,7 \mathrm{~b}$ \\
\hline $\mathrm{LGS}_{4} \times \mathrm{LGS}_{6}$ & $9,51 \mathrm{~b}$ & $5,34 \mathrm{~b}$ & $4,87 \mathrm{a}$ & $5,47 \mathrm{a}$ & $242 a$ & $163 b$ & $180 \mathrm{a}$ & $176 \mathrm{~b}$ & $137 \mathrm{~b}$ & $77 \mathrm{a}$ & $85 a$ & $100 \mathrm{a}$ & $4,0 \mathrm{e}$ & $3,0 \mathrm{~b}$ & $4,7 \mathrm{~b}$ & $4,0 \mathrm{~d}$ & $4,0 \mathrm{a}$ \\
\hline $\mathrm{LGS}_{4} \times \mathrm{XGS}_{8}$ & $8,24 \mathrm{c}$ & $5,16 \mathrm{~b}$ & $5,56 \mathrm{a}$ & $5,61 \mathrm{a}$ & $240 \mathrm{~b}$ & $175 \mathrm{a}$ & $188 \mathrm{a}$ & $149 \mathrm{e}$ & $138 \mathrm{~b}$ & $78 \mathrm{a}$ & $97 \mathrm{a}$ & $66 \mathrm{~d}$ & $2,3 \mathrm{f}$ & $4,3 \mathrm{a}$ & $4,3 b$ & $4,0 \mathrm{~d}$ & $2,7 \mathrm{~b}$ \\
\hline $\mathrm{LGS}_{4} \times \mathrm{LGS}_{9}$ & $8,64 \mathrm{c}$ & $5,83 \mathrm{a}$ & $5,54 \mathrm{a}$ & $6,59 \mathrm{a}$ & $258 \mathrm{a}$ & $167 \mathrm{~b}$ & $192 \mathrm{a}$ & $177 \mathrm{~b}$ & $147 \mathrm{a}$ & $78 \mathrm{a}$ & $100 \mathrm{a}$ & $93 b$ & $3,0 \mathrm{f}$ & $3,5 \mathrm{a}$ & $4,0 \mathrm{c}$ & $5,7 \mathrm{c}$ & $4,7 \mathrm{a}$ \\
\hline $\mathrm{LGS}_{4} \times \mathrm{LGS}_{10}$ & $8,48 \mathrm{c}$ & $5,53 \mathrm{a}$ & $5,39 a$ & $6,02 \mathrm{a}$ & $228 b$ & $167 \mathrm{~b}$ & $188 \mathrm{a}$ & $186 a$ & $127 \mathrm{~b}$ & $68 \mathrm{~b}$ & $103 a$ & $103 a$ & $3,5 \mathrm{e}$ & $3,2 b$ & $5,5 \mathrm{a}$ & $5,0 \mathrm{c}$ & $4,7 \mathrm{a}$ \\
\hline $\mathrm{LGS}_{5} \times \mathrm{xGS}_{6}$ & $7,11 \mathrm{c}$ & $4,98 \mathrm{~b}$ & $5,24 \mathrm{a}$ & $4,45 \mathrm{a}$ & $232 b$ & $165 \mathrm{~b}$ & $188 \mathrm{a}$ & $158 \mathrm{~d}$ & $132 \mathrm{~b}$ & $72 b$ & $92 \mathrm{a}$ & $75 \mathrm{c}$ & $5,3 \mathrm{c}$ & $4,0 \mathrm{a}$ & $4,7 \mathrm{~b}$ & $5,3 \mathrm{c}$ & $5,0 \mathrm{a}$ \\
\hline $\mathrm{LGS}_{5} \mathrm{xLGS}_{7}$ & $7,39 \mathrm{c}$ & $4,98 \mathrm{~b}$ & $4,91 \mathrm{a}$ & $4,36 \mathrm{a}$ & $238 b$ & $178 \mathrm{a}$ & $190 \mathrm{a}$ & $175 b$ & $137 \mathrm{~b}$ & $78 \mathrm{a}$ & $95 \mathrm{a}$ & $88 b$ & $6,3 b$ & $3,5 \mathrm{a}$ & $5,0 \mathrm{~b}$ & $4,7 d$ & $3,0 \mathrm{~b}$ \\
\hline $\mathrm{LGS}_{5} \times \mathrm{LGS}_{8}$ & $8,10 \mathrm{c}$ & $4,67 b$ & $6,88 \mathrm{a}$ & $5,63 \mathrm{a}$ & $252 a$ & $180 \mathrm{a}$ & $193 a$ & $178 b$ & $138 \mathrm{~b}$ & $72 b$ & $92 a$ & $90 \mathrm{~b}$ & $3,0 \mathrm{f}$ & $2,8 \mathrm{~b}$ & $4,7 \mathrm{~b}$ & $6,0 \mathrm{~b}$ & $5,0 \mathrm{a}$ \\
\hline $\mathrm{LGS}_{5} \times \mathrm{LGS}_{9}$ & $8,57 \mathrm{c}$ & $5,72 \mathrm{a}$ & $5,29 a$ & $6,74 a$ & $235 b$ & $160 \mathrm{~b}$ & $193 a$ & $158 \mathrm{~d}$ & $132 \mathrm{~b}$ & $65 b$ & $92 a$ & $93 b$ & $3,0 \mathrm{f}$ & $3,0 \mathrm{~b}$ & $4,7 \mathrm{~b}$ & $5,7 \mathrm{c}$ & $5,0 \mathrm{a}$ \\
\hline $\operatorname{LGS}_{5} x \mathrm{LGS}_{10}$ & $7,14 \mathrm{c}$ & $4,89 \mathrm{~b}$ & $5,05 \mathrm{a}$ & $6,10 \mathrm{a}$ & $225 b$ & $158 \mathrm{~b}$ & $189 \mathrm{a}$ & $178 \mathrm{~b}$ & $113 \mathrm{c}$ & $68 \mathrm{~b}$ & $82 a$ & $85 \mathrm{c}$ & $2,8 \mathrm{f}$ & $3,2 b$ & $5,0 \mathrm{~b}$ & $5,0 \mathrm{c}$ & $5,2 \mathrm{a}$ \\
\hline $\mathrm{LGS}_{6} \mathrm{xLGS}_{7}$ & $6,41 \mathrm{c}$ & $4,30 \mathrm{~b}$ & $5,68 \mathrm{a}$ & $6,47 \mathrm{a}$ & $252 a$ & $180 \mathrm{a}$ & $192 a$ & $179 b$ & $148 \mathrm{a}$ & $93 a$ & $95 \mathrm{a}$ & $90 \mathrm{~b}$ & $5,3 \mathrm{c}$ & $4,0 \mathrm{a}$ & $5,7 \mathrm{a}$ & $4,5 \mathrm{~d}$ & $4,5 \mathrm{a}$ \\
\hline $\mathrm{LGS}_{6} \times \mathrm{LGS}_{8}$ & $6,56 \mathrm{c}$ & $4,78 b$ & $6,84 \mathrm{a}$ & $6,41 \mathrm{a}$ & $232 b$ & $170 \mathrm{~b}$ & $187 \mathrm{a}$ & $172 \mathrm{c}$ & $132 \mathrm{~b}$ & $75 a$ & $98 \mathrm{a}$ & $78 \mathrm{c}$ & $3,3 \mathrm{f}$ & $2,8 \mathrm{~b}$ & $5,0 \mathrm{~b}$ & $5,0 \mathrm{c}$ & $3,2 \mathrm{~b}$ \\
\hline $\mathrm{LGS}_{6} \mathrm{xLGS}_{9}$ & $8,52 \mathrm{c}$ & $6,02 a$ & $6,82 \mathrm{a}$ & $6,76 a$ & $237 b$ & $172 b$ & $182 \mathrm{a}$ & $173 b$ & $148 \mathrm{a}$ & $80 a$ & $102 a$ & $82 \mathrm{c}$ & $5,0 \mathrm{~d}$ & $3,5 \mathrm{a}$ & $4,0 \mathrm{c}$ & $6,0 \mathrm{~b}$ & $5,2 \mathrm{a}$ \\
\hline $\mathrm{LGS}_{6} \times \mathrm{LGS}_{10}$ & $7,42 \mathrm{c}$ & $5,21 \mathrm{~b}$ & $6,18 \mathrm{a}$ & $6,61 \mathrm{a}$ & $247 \mathrm{a}$ & $157 \mathrm{~b}$ & $202 a$ & $171 \mathrm{c}$ & $138 \mathrm{~b}$ & $70 \mathrm{~b}$ & $95 \mathrm{a}$ & $98 \mathrm{a}$ & $3,0 \mathrm{f}$ & $3,5 \mathrm{a}$ & $5,0 \mathrm{~b}$ & $6,0 \mathrm{~b}$ & $5,5 \mathrm{a}$ \\
\hline $\mathrm{LGS}_{7} \mathrm{XLGS}_{8}$ & $7,68 \mathrm{c}$ & $3,23 b$ & $5,96 \mathrm{a}$ & $6,35 a$ & $255 \mathrm{a}$ & $165 \mathrm{~b}$ & $188 \mathrm{a}$ & $175 b$ & $133 b$ & $72 b$ & $106 a$ & $88 \mathrm{~b}$ & $6,8 b$ & $3,8 \mathrm{a}$ & $4,0 \mathrm{c}$ & $4,0 \mathrm{~d}$ & $4,2 \mathrm{a}$ \\
\hline $\mathrm{LGS}_{7} \times \mathrm{LGS}_{9}$ & $7,99 \mathrm{c}$ & $5,14 \mathrm{~b}$ & $6,01 \mathrm{a}$ & $5,18 \mathrm{a}$ & $243 a$ & $177 \mathrm{a}$ & $187 \mathrm{a}$ & $178 b$ & $138 \mathrm{~b}$ & $82 a$ & $88 a$ & $88 \mathrm{~b}$ & $4,3 \mathrm{e}$ & $3,7 \mathrm{a}$ & $5,7 \mathrm{a}$ & $3,7 \mathrm{e}$ & $3,0 \mathrm{~b}$ \\
\hline $\mathrm{LGS}_{7} \times \mathrm{LGS}_{10}$ & $8,11 \mathrm{c}$ & $4,93 \mathrm{~b}$ & $4,88 \mathrm{a}$ & $5,39 a$ & $232 b$ & $173 a$ & $177 \mathrm{a}$ & $183 a$ & $133 b$ & $82 a$ & $88 \mathrm{a}$ & $85 \mathrm{c}$ & $6,0 \mathrm{c}$ & $4,0 \mathrm{a}$ & $4,7 \mathrm{~b}$ & $4,0 \mathrm{~d}$ & $3,8 \mathrm{~b}$ \\
\hline $\mathrm{LGS}_{8} \times \mathrm{LGS}_{9}$ & $7,67 \mathrm{c}$ & $4,13 b$ & $5,87 \mathrm{a}$ & $5,84 a$ & $258 \mathrm{a}$ & $168 \mathrm{~b}$ & $188 \mathrm{a}$ & $175 b$ & $148 \mathrm{a}$ & $77 \mathrm{a}$ & $110 \mathrm{a}$ & $95 \mathrm{~b}$ & $3,5 \mathrm{e}$ & $4,0 \mathrm{a}$ & $4,0 \mathrm{c}$ & $5,8 \mathrm{~b}$ & $5,0 \mathrm{a}$ \\
\hline $\mathrm{LGS}_{8} x \mathrm{LGS}_{10}$ & $7,81 \mathrm{c}$ & $5,39 b$ & $5,05 \mathrm{a}$ & $4,73 a$ & $230 \mathrm{~b}$ & $162 b$ & $187 \mathrm{a}$ & $160 \mathrm{~d}$ & $120 \mathrm{c}$ & $72 b$ & $95 \mathrm{a}$ & $80 \mathrm{c}$ & $2,5 \mathrm{f}$ & $1,7 \mathrm{c}$ & $4,0 \mathrm{c}$ & $5,0 \mathrm{c}$ & $5,0 \mathrm{a}$ \\
\hline$\underline{L G S}_{9} \mathrm{XGS}_{10}$ & $6,83 \mathrm{c}$ & $5,06 \mathrm{~b}$ & $6,37 \mathrm{a}$ & $5,39 a$ & $230 \mathrm{~b}$ & $163 \mathrm{~b}$ & $195 \mathrm{a}$ & $168 \mathrm{c}$ & $122 \mathrm{c}$ & $65 \mathrm{~b}$ & $102 \mathrm{a}$ & $93 \mathrm{~b}$ & $4,3 \mathrm{e}$ & $4,0 \mathrm{a}$ & $5,7 \mathrm{a}$ & $4,0 \mathrm{~d}$ & $3,0 \mathrm{~b}$ \\
\hline $\mathrm{P} 3069^{(2)}$ & $5,94 \mathrm{c}$ & $5,04 \mathrm{~b}$ & $4,75 \mathrm{a}$ & $5,91 \mathrm{a}$ & $222 b$ & $160 \mathrm{~b}$ & $184 \mathrm{a}$ & $177 \mathrm{~b}$ & $112 \mathrm{c}$ & $62 b$ & $87 \mathrm{a}$ & $87 \mathrm{~b}$ & $4,0 \mathrm{e}$ & $4,2 \mathrm{a}$ & $4,0 \mathrm{c}$ & $8,0 \mathrm{a}$ & $7,0 \mathrm{a}$ \\
\hline P30F90 ${ }^{(2)}$ & $12,11 \mathrm{a}$ & $6,63 a$ & $5,60 \mathrm{a}$ & $5,81 \mathrm{a}$ & $247 \mathrm{a}$ & $187 \mathrm{a}$ & $183 a$ & $167 \mathrm{c}$ & $138 \mathrm{~b}$ & $73 b$ & $92 \mathrm{a}$ & $92 b$ & $3,0 \mathrm{f}$ & $4,0 \mathrm{a}$ & $3,0 \mathrm{~d}$ & $4,0 \mathrm{~d}$ & $3,0 \mathrm{~b}$ \\
\hline BG 7060 (2) & $8,73 \mathrm{c}$ & $5,61 \mathrm{a}$ & $5,35 \mathrm{a}$ & $6,04 a$ & $245 \mathrm{a}$ & $170 \mathrm{~b}$ & $193 a$ & $169 \mathrm{c}$ & $147 \mathrm{a}$ & $75 \mathrm{a}$ & $103 a$ & $91 b$ & $2,7 \mathrm{f}$ & $3,5 \mathrm{a}$ & $4,0 \mathrm{c}$ & $6,3 b$ & $4,0 \mathrm{a}$ \\
\hline Balu $761^{(2)}$ & $9,06 \mathrm{~b}$ & $4,98 \mathrm{~b}$ & $4,78 \mathrm{a}$ & $5,21 \mathrm{a}$ & $223 b$ & $172 b$ & $188 \mathrm{a}$ & $181 \mathrm{a}$ & $133 b$ & $85 a$ & $85 \mathrm{a}$ & $105 \mathrm{a}$ & $4,5 \mathrm{~d}$ & $3,0 \mathrm{~b}$ & $4,0 \mathrm{c}$ & $4,7 d$ & $4,0 \mathrm{a}$ \\
\hline Dow $2 \mathrm{~A} 120^{(2)}$ & $7,01 \mathrm{c}$ & $5,14 \mathrm{~b}$ & $5,85 \mathrm{a}$ & $4,26 \mathrm{a}$ & $235 \mathrm{~b}$ & $167 \mathrm{~b}$ & $187 \mathrm{a}$ & $171 \mathrm{c}$ & $110 \mathrm{c}$ & $58 \mathrm{~b}$ & $98 \mathrm{a}$ & $88 \mathrm{~b}$ & $8,2 \mathrm{a}$ & $3,8 \mathrm{a}$ & $5,7 \mathrm{a}$ & $6,7 \mathrm{~b}$ & $5,0 \mathrm{a}$ \\
\hline Média Testemunhas & 8,57 & 5,48 & 5,27 & 5,45 & 234 & 171 & 187 & 173 & 128 & 71 & 93 & 93 & 4,5 & 3,7 & 4,1 & 5,9 & 4,6 \\
\hline Média Hibridos & 8,55 & 5,29 & 5,73 & 5,78 & 242 & 170 & 189 & 171 & 137 & 74 & 96 & 87 & 4,3 & 3,5 & 4,6 & 4,5 & 3,8 \\
\hline Média Geral & 8,56 & 5,31 & 5,68 & 5,75 & 241 & 170 & 189 & 172 & 136 & 74 & 95 & 88 & 4,3 & 3,5 & 4,6 & 4,7 & 3,9 \\
\hline
\end{tabular}

${ }^{(1)}$ Médias seguidas de letras iguais não diferem entre si, pelo teste de Scott \& Knott, a 5\% de probabilidade. ${ }^{(2)}$ Híbridos comerciais, testemunhas. 
quando em cruzamento com os demais genitores e, ao se considerar a interação com local, mostra ainda que as estimativas de CGC $\left(\hat{\mathrm{g}}_{\mathrm{i}}\right)$ foram diferenciadas nesses locais e que é necessário que a seleção seja realizada em cada local. Estudos sobre resistência a doenças e quanto à produtividade mostram a existência de interações de CGC e CEC com o ambiente (Paterniani et al., 2000; Carson, 2001; Lopes et al., 2007; Guimarães et al., 2009; Vivek et al., 2010).

As estimativas de $\mathrm{g}_{\mathrm{i}} \mathrm{s}$, com valores dentro do intervalo zero mais ou menos o desvio-padrão da estimativa de $g_{i}$ [DP $\left(\mathrm{g}_{\mathrm{i}}\right)$ ], para cada característica, foram consideradas nulas, e indicam que os genitores não contribuíram significativamente para o aumento ou redução do caráter, quando em cruzamento com os demais genitores. Para Cruz et al. (2004), valores elevados para CGC, positivos ou negativos, mostram que o genitor é muito superior ou inferior, respectivamente, aos demais genitores do dialelo. Neste contexto, as linhagens com melhores $\hat{g}_{\mathrm{i}} \mathrm{s}$ quanto à produtividade e demais características foram $\mathrm{LGS}_{2}, \mathrm{LGS}_{9}, \mathrm{LGS}_{4}$ e $\mathrm{LGS}_{3}$, presentes na composição dos híbridos mais promissores. Com base nos locais, as linhagens $\mathrm{LGS}_{2}$ e $\mathrm{LGS}_{9}$ apresentaram as melhores estimativas de $\hat{\mathrm{g}}_{\mathrm{i}}$ 's quanto à produtividade e resistência à mancha-branca. A linhagem $\mathrm{LGS}_{4}$ apresentou as melhores estimativas de $\hat{\mathrm{g}}_{\mathrm{i}}$ 's quanto à severidade de todas as doenças estudadas, e a $\mathrm{LGS}_{3}$ destacou-se por apresentar estimativas que contribuíram para reduzir a severidade de helmintosporiose, ferrugem tropical, ferrugem-polissora e as médias de altura da planta e altura de espiga (Tabela 3 ).

Os efeitos de capacidade específica de combinação (CEC) também foram significativos para todas as características, bem como o de suas interações com locais, nas análises conjuntas (Tabela 1). Os efeitos de CEC indicam que determinadas combinações híbridas exibem oscilação em seu desempenho que não pode ser explicada pela média dos pais e suas capacidades gerais de combinação (Cruz et al., 2004). A significância da interação mostra que houve comportamento diferenciado dos genótipos em cada local, quanto ao desempenho dos híbridos e à capacidade combinatória das linhagens avaliadas.

Considerando-se todos os locais de avaliação, as melhores estimativas de $\mathrm{CEC}\left(\hat{\mathrm{s}}_{\mathrm{ii}}\right)$ quanto à produtividade ficaram por conta das combinações entre $\mathrm{LGS}_{2} \mathrm{xLGS}_{6}$ e $\mathrm{LGS}_{3} \mathrm{XLGS}_{9}$, com valores muito superiores em Pitangueiras, onde foram encontradas as maiores médias (Tabela 4). Quanto às características altura de planta e de espiga, destacaram-se as combinações específicas entre $\mathrm{LGS}_{1} \mathrm{xLGS}$ e $\mathrm{LGS}_{4} \mathrm{xLGS}$. As estimativas de $\mathrm{s}_{\mathrm{ii}}$ indicam cruzamentos promissores com relação à resistência às doenças foliares avaliadas, com destaque para as combinações: $\mathrm{LGS}_{4} \mathrm{xLGS}$ e $\mathrm{LGS}_{6} \mathrm{xLGS}_{9}$,

Tabela 3. Estimativas das médias dos cruzamentos ( $m$ ), de CGC ( $\left.\mathrm{g}_{\mathrm{i}}\right)$ e do desvio-padrão (DP) das estimativas, e contrastes entre os genitores, quanto às características produtividade de grãos $\left(\mathrm{Mg} \mathrm{ha}^{-1}\right)$, altura de planta $(\mathrm{cm})$ e altura de espiga $(\mathrm{cm})$; notas de severidade de mancha-branca (MB), helmintosporiose (ET), ferrugem tropical (PHY) e ferrugem-polissora (FP), avaliadas em Pitangueiras (PIT), Sorriso (SO), Jaciara (JA) e Dourados (DO).

\begin{tabular}{|c|c|c|c|c|c|c|c|c|c|c|c|c|c|c|c|c|c|}
\hline \multirow[t]{2}{*}{ Estimativa $^{(1)}$} & \multicolumn{4}{|c|}{ Produtividade } & \multicolumn{4}{|c|}{ Altura de planta } & \multicolumn{4}{|c|}{ Altura de espiga } & \multicolumn{2}{|c|}{ MB } & \multirow{2}{*}{$\begin{array}{l}\text { ET } \\
\text { PIT }\end{array}$} & \multirow{2}{*}{$\begin{array}{c}\text { PHY } \\
\text { PIT }\end{array}$} & \multirow{2}{*}{$\begin{array}{l}\text { FP } \\
\text { PIT } \\
\end{array}$} \\
\hline & PIT & $\mathrm{SO}$ & JA & DO & PIT & $\mathrm{SO}$ & JA & $\mathrm{DO}$ & PIT & SO & JA & $\mathrm{DO}$ & PIT & $\mathrm{SO}$ & & & \\
\hline $\mathrm{m}$ & 8,56 & 5,24 & 5,75 & 5,78 & 242 & 171 & 189 & 171 & 137 & 74 & 96 & 87 & 4,3 & 3,5 & 4,6 & 4,5 & 3,8 \\
\hline$g_{1}$ & 0,11 & 0,28 & 0,21 & $-0,26$ & 5 & 4 & 1 & -2 & 4 & -1 & 1 & -3 & 0,7 & 0,7 & 0,1 & $-0,5$ & $-0,7$ \\
\hline $\mathrm{g}_{2}$ & 1,44 & 0,44 & 0,20 & $-0,10$ & 15 & 8 & 0 & -1 & 15 & 7 & 1 & 2 & $-1,0$ & $-0,3$ & 0,0 & 0,1 & 0,4 \\
\hline $\mathrm{g}_{3}$ & 0,61 & $-0,19$ & $-0,08$ & 0,00 & -11 & -6 & 3 & 1 & -12 & -8 & 0 & -3 & 1,1 & $-0,1$ & $-0,2$ & $-0,6$ & $-1,1$ \\
\hline $\mathrm{g}_{4}$ & 0,62 & 0,06 & $-0,52$ & 0,23 & -1 & -1 & -4 & 1 & 1 & 0 & -1 & 8 & $-0,7$ & 0,0 & $-0,5$ & $-0,3$ & $-0,1$ \\
\hline $\mathrm{g}_{5}$ & $-0,35$ & 0,05 & $-0,35$ & $-0,10$ & -6 & -4 & -1 & -1 & -8 & -6 & -3 & 2 & $-0,5$ & $-0,3$ & $-0,2$ & 0,5 & 0,3 \\
\hline $\mathrm{g}_{6}$ & $-0,43$ & 0,14 & 0,15 & 0,16 & -4 & 0 & 2 & -1 & 3 & 4 & -1 & -1 & 0,2 & 0,1 & 0,4 & 0,3 & 0,6 \\
\hline$g_{7}$ & $-0,80$ & $-0,46$ & $-0,11$ & 0,16 & 4 & 7 & 0 & 6 & 4 & 9 & -1 & 2 & 1,8 & 0,2 & 0,3 & $-0,5$ & $-0,2$ \\
\hline $\mathrm{g}_{8}$ & $-0,63$ & $-0,72$ & 0,39 & $-0,09$ & 5 & 1 & 0 & -4 & -1 & -1 & 5 & -6 & $-0,7$ & $-0,2$ & $-0,4$ & 0,3 & 0,1 \\
\hline $\mathrm{g}_{9}$ & $-0,02$ & 0,42 & 0,25 & 0,09 & 0 & -3 & 0 & 1 & 1 & 0 & 1 & 0 & $-0,6$ & 0,2 & 0,0 & 0,4 & 0,1 \\
\hline$\underline{g_{10}}$ & $-0,56$ & $-0,04$ & $-0,13$ & $-0,10$ & -7 & -6 & 0 & 1 & -8 & -5 & -1 & 1 & $-0,4$ & $-0,2$ & 0,4 & 0,3 & 0,5 \\
\hline $\mathrm{DP}\left(\mathrm{g}_{\mathrm{i}}\right)$ & 0,20 & 0,12 & 0,20 & 0,16 & 2 & 2 & 2 & 1 & 2 & 2 & 2 & 1 & 0,1 & 0,1 & 0,1 & 0,1 & 0,2 \\
\hline$\underline{D P}\left(g_{i}-g_{i}\right)$ & 0,30 & 0,18 & 0,30 & 0,24 & 3 & 3 & 3 & 2 & 3 & 2 & 4 & 2 & 0,1 & 0,2 & 0,2 & 0,2 & 0,2 \\
\hline
\end{tabular}

${ }^{(1)} \mathrm{g}_{1}$ a $\mathrm{g}_{10}$, efeito da capacidade geral de combinação nas linhagens 1 a $10 ; \mathrm{g}_{\mathrm{i}}$ e $\mathrm{g}_{\mathrm{i}}$, efeitos da capacidade geral de combinação (CGC) da i-ésima linhagem $\mathrm{LGS}_{\mathrm{i}}$ e i'-ésima linhagem $\mathrm{LGS}_{\mathrm{i}}$, na combinação híbrida entre linhagens $\mathrm{LGS}_{\mathrm{i}} \mathrm{xLGS} \mathrm{LG}_{\mathrm{i}} ; \mathrm{DP}\left(\mathrm{g}_{\mathrm{i}}\right)$, desvio-padrão das estimativas CGC; DP $\left(\mathrm{g}_{\mathrm{i}}\right.$ - $\left.\mathrm{g}_{\mathrm{i}}\right)$, desvio-padrão dos contrastes das estimativas de CGC. 
Tabela 4. Estimativas do efeito da capacidade específica de combinação (CEC) entre os genitores i e i'( $\left(_{\text {iii' }}\right.$ ) e desvio-padrão (DP) das estimativas CEC e de seus contrastes, nas combinações dos genitores dois a dois, para as características produtividade de grãos, $\left(\mathrm{Mg} \mathrm{ha}^{-1}\right)$, altura de planta e altura de espiga $(\mathrm{cm})$; notas de severidade de mancha-branca $(\mathrm{MB})$, helmintosporiose (ET), ferrugem tropical (PHY) e ferrugem-polissora (FP), avaliadas em Pitangueiras (PIT), Sorriso (SO), Jaciara (JA) e Dourados (DO).

\begin{tabular}{|c|c|c|c|c|c|c|c|c|c|c|c|c|c|c|c|c|c|}
\hline \multirow[t]{2}{*}{$\mathrm{S}_{\mathrm{i} i}$} & \multicolumn{4}{|c|}{ Produtividade } & \multicolumn{4}{|c|}{ Altura de planta } & \multicolumn{4}{|c|}{ Altura de espiga } & \multicolumn{2}{|c|}{ MB } & \multirow{2}{*}{$\begin{array}{l}\text { ET } \\
\text { PIT }\end{array}$} & \multirow{2}{*}{$\begin{array}{c}\text { PHY } \\
\text { PIT }\end{array}$} & \multirow{2}{*}{$\begin{array}{l}\text { FP } \\
\text { PIT }\end{array}$} \\
\hline & PIT & $\mathrm{SO}$ & JA & DO & PIT & $\mathrm{SO}$ & $\mathrm{JA}$ & $\mathrm{DO}$ & PIT & SO & JA & $\mathrm{DO}$ & PIT & $\mathrm{SO}$ & & & \\
\hline $\mathrm{s}_{12}$ & $-0,40$ & $-0,38$ & $-0,06$ & $-0,89$ & 3 & 6 & 2 & 3 & -2 & -4 & 10 & 2 & $-0,5$ & $-0,4$ & $-0,2$ & $-0,8$ & $-0,5$ \\
\hline $\mathrm{s}_{13}$ & $-0,91$ & $-0,46$ & $-1,13$ & 0,34 & 12 & 5 & -9 & 4 & 14 & 8 & -6 & 7 & 0,9 & 0,5 & 0,2 & 0,1 & 0,4 \\
\hline $\mathrm{s}_{14}$ & 0,97 & $-1,08$ & $-0,30$ & $-0,11$ & 4 & -8 & 3 & 0 & 6 & -8 & -3 & 5 & 1,4 & 0,1 & $-0,4$ & $-0,7$ & 0,4 \\
\hline $\mathrm{S}_{15}$ & 1,05 & 0,46 & 0,30 & 0,26 & 2 & -2 & 4 & 13 & 2 & -3 & 4 & 16 & $-0,5$ & $-0,7$ & 0,5 & 0,5 & 0,0 \\
\hline $\mathrm{s}_{16}$ & $-0,74$ & 0,47 & $-0,78$ & 0,13 & -17 & 1 & 2 & 0 & -10 & 3 & -3 & 7 & $-0,2$ & 0,4 & $-0,1$ & $-0,8$ & $-0,4$ \\
\hline $\mathrm{s}_{17}$ & $-0,17$ & 0,48 & 0,75 & 0,12 & -5 & 2 & 2 & -12 & -4 & -3 & -4 & -11 & $-0,8$ & 0,4 & 0,0 & 1,2 & $-0,6$ \\
\hline $\mathrm{s}_{19}$ & $-1,10$ & 0,73 & 0,10 & $-0,06$ & -9 & -5 & 1 & -6 & -4 & 3 & 3 & -21 & $-0,8$ & $-0,7$ & $-0,2$ & 0,3 & 0,2 \\
\hline $\mathrm{s}_{110}$ & 1,31 & $-0,21$ & 1,13 & 0,21 & 10 & 1 & -5 & -3 & -2 & 4 & -1 & -7 & 0,4 & 0,3 & 0,3 & 0,2 & 0,4 \\
\hline $\mathrm{S}_{23}$ & $-0,22$ & $-0,42$ & 0,63 & $-0,49$ & -9 & -12 & 1 & 11 & -16 & -2 & 2 & 10 & 0,0 & $-0,5$ & 0,2 & 1,0 & 0,4 \\
\hline $\mathrm{s}_{24}$ & 0,03 & $-0,02$ & $-0,21$ & 0,76 & -2 & 6 & -2 & -8 & 1 & 1 & -4 & -1 & $-0,2$ & $-0,3$ & 0,4 & 0,7 & 0,2 \\
\hline $\mathrm{s}_{25}$ & $-1,07$ & $-0,27$ & 1,02 & 0,18 & 4 & -13 & -4 & -11 & 4 & -6 & 1 & -15 & 0,1 & 0,5 & 0,1 & $-0,1$ & $-1,0$ \\
\hline $\mathrm{S}_{26}$ & 2,02 & 0,76 & 0,25 & $-0,22$ & 7 & 8 & 8 & -6 & 9 & 1 & 5 & -5 & 0,4 & $-0,4$ & 0,0 & $-0,4$ & 0,9 \\
\hline $\mathrm{S}_{27}$ & 0,26 & 0,29 & $-0,46$ & 1,41 & 7 & 4 & 4 & 13 & 6 & 7 & -1 & 0 & $-0,2$ & 0,4 & 0,1 & 0,4 & 1,2 \\
\hline $\mathrm{S}_{29}$ & $-0,25$ & 0,09 & $-0,92$ & $-0,92$ & -10 & -4 & -6 & 0 & -16 & -1 & -15 & 6 & 0,2 & 0,7 & $-0,1$ & $-0,5$ & $-1,1$ \\
\hline $\mathrm{S}_{210}$ & $-0,37$ & $-0,06$ & $-0,27$ & 0,17 & 0 & 5 & -3 & -3 & 14 & 3 & 1 & 2 & 0,1 & $-0,1$ & $-0,4$ & $-0,2$ & $-0,1$ \\
\hline $\mathrm{S}_{34}$ & $-0,64$ & 0,09 & 0,81 & $-0,52$ & -5 & -2 & 6 & -6 & -3 & 3 & -3 & -8 & $-0,8$ & $-0,3$ & $-0,6$ & 0,2 & 0,0 \\
\hline $\mathrm{s}_{35}$ & 0,48 & 0,22 & $-1,06$ & 0,67 & -3 & 3 & -2 & -5 & -7 & 3 & -1 & -7 & $-0,6$ & $-0,4$ & $-0,7$ & $-0,4$ & $-0,1$ \\
\hline $\mathrm{s}_{36}$ & 0,23 & $-0,20$ & $-0,19$ & $-0,24$ & -4 & 2 & -2 & 2 & -5 & -3 & 2 & -5 & $-0,3$ & 0,8 & 0,9 & $-0,2$ & $-0,4$ \\
\hline$s_{37}$ & $-0,83$ & 0,45 & 0,78 & 0,37 & -2 & -2 & 7 & -10 & 2 & -1 & 6 & 12 & 0,8 & $-0,5$ & $-0,2$ & $-0,4$ & 0,5 \\
\hline $\mathrm{S}_{39}$ & 2,22 & 0,33 & 0,37 & $-0,04$ & 3 & 5 & -3 & 11 & 4 & -1 & -3 & -2 & $-0,4$ & 0,2 & 0,1 & $-0,8$ & 0,1 \\
\hline $\mathrm{S}_{310}$ & $-0,32$ & $-0,02$ & $-0,22$ & $-0,1$ & 9 & 2 & 2 & -6 & 11 & -7 & 4 & -6 & 0,3 & 0,2 & 0,2 & 0,5 & $-0,9$ \\
\hline $\mathrm{S}_{45}$ & $-0,16$ & 0,18 & $-0,07$ & 0,26 & -7 & 0 & -12 & 10 & -4 & -1 & 8 & 14 & $-0,1$ & 0,0 & $-0,9$ & $-0,7$ & $-0,4$ \\
\hline $\mathrm{S}_{46}$ & 0,77 & $-0,11$ & $-0,51$ & $-0,7$ & 4 & -6 & -7 & 5 & -4 & -2 & -9 & 7 & 0,2 & 1,1 & 0,2 & $-0,5$ & $-0,4$ \\
\hline $\mathrm{S}_{48}$ & $-0,31$ & 0,57 & $-0,06$ & $-0,31$ & -6 & 5 & 3 & -18 & 1 & 4 & -3 & -22 & $-0,6$ & $-0,3$ & 0,6 & $-0,6$ & $-1,2$ \\
\hline $\mathrm{s}_{49}$ & $-0,51$ & 0,10 & 0,06 & 0,49 & 17 & 1 & 7 & 4 & 8 & 4 & 4 & -1 & $-0,1$ & 0,0 & $-0,1$ & 1,1 & 0,8 \\
\hline $\mathrm{S}_{410}$ & $-0,13$ & 0,27 & 0,28 & 0,12 & -6 & 3 & 3 & 13 & -4 & -2 & 10 & 7 & 0,3 & $-0,3$ & 0,9 & 0,5 & 0,5 \\
\hline $\mathbf{S}_{56}$ & $-0,66$ & $-0,46$ & $-0,31$ & $-1,39$ & -1 & -1 & -1 & -11 & 0 & 0 & 0 & -12 & 1,3 & $-0,7$ & $-0,1$ & 0,0 & 0,2 \\
\hline $\mathrm{S}_{57}$ & $-0,02$ & 0,14 & $-0,38$ & $-1,48$ & -2 & 5 & 2 & -2 & 3 & 1 & 4 & -3 & 0,7 & 0,1 & 0,3 & 0,1 & $-0,9$ \\
\hline $\mathrm{s}_{58}$ & 0,52 & 0,09 & 1,08 & 0,04 & 11 & 13 & 6 & 12 & 10 & 4 & -6 & 7 & $-0,1$ & $-0,1$ & 0,6 & 0,6 & 0,7 \\
\hline $\mathrm{S}_{59}$ & 0,38 & 0,01 & $-0,37$ & 0,96 & -1 & -3 & 6 & -13 & 1 & -3 & -2 & 4 & $-0,3$ & 0,7 & 0,2 & 0,3 & 0,7 \\
\hline $\mathrm{S}_{510}$ & $-0,51$ & $-0,36$ & $-0,22$ & 0,52 & -4 & -2 & 1 & 7 & -8 & 5 & -9 & -5 & $-0,6$ & 0,5 & 0,1 & $-0,3$ & 0,6 \\
\hline $\mathrm{s}_{67}$ & $-0,92$ & $-0,64$ & $-0,10$ & 0,37 & 9 & 2 & 1 & 2 & 4 & 6 & 1 & 2 & $-1,0$ & $-0,4$ & 0,4 & 0,2 & 0,3 \\
\hline $\mathrm{S}_{68}$ & $-0,94$ & 0,11 & 0,55 & 0,56 & -11 & -1 & -4 & 5 & -7 & -2 & -2 & -2 & $-0,5$ & 0,0 & 0,3 & $-0,1$ & $-1,4$ \\
\hline $\mathrm{s}_{69}$ & 0,41 & 0,21 & 0,67 & 0,73 & -2 & 4 & -9 & 2 & 8 & 2 & 5 & -4 & 1,1 & $-0,7$ & $-1,0$ & 0,8 & 0,6 \\
\hline $\mathrm{s}_{610}$ & $-0,16$ & $-0,14$ & 0,41 & 0,77 & 15 & -8 & 11 & 0 & 6 & -4 & 1 & 11 & $-1,1$ & $-0,3$ & $-0,4$ & 0,9 & 0,6 \\
\hline $\mathbf{s}_{78}$ & 0,54 & $-0,84$ & $-0,08$ & 0,50 & 4 & -13 & -1 & 2 & -7 & -11 & 7 & 5 & 1,4 & 0,1 & $-0,6$ & $-0,4$ & 0,4 \\
\hline $\mathbf{S}_{79}$ & 0,25 & $-0,07$ & 0,12 & $-0,85$ & -3 & 2 & -2 & 0 & -4 & -2 & -7 & -1 & $-1,2$ & 0,5 & 0,8 & $-0,7$ & $-0,7$ \\
\hline $\mathrm{S}_{710}$ & 0,90 & 0,19 & $-0,64$ & $-0,44$ & -8 & 1 & -13 & 5 & 0 & 3 & -5 & -5 & 0,3 & $-0,5$ & $-0,7$ & $-0,3$ & $-0,2$ \\
\hline $\mathrm{S}_{89}$ & $-0,24$ & $-0,82$ & $-0,53$ & 0,06 & 11 & 0 & -1 & 7 & 11 & 3 & 8 & 14 & 0,5 & $-0,4$ & $-0,3$ & 0,6 & 0,9 \\
\hline $\mathrm{S}_{810}$ & 0,43 & 0,90 & $-0,97$ & $-0,86$ & -10 & -4 & -3 & -8 & -8 & 3 & -5 & -2 & $-0,7$ & 0,6 & $-0,7$ & $-0,1$ & 0,6 \\
\hline $\mathrm{S}_{910}$ & $-1,15$ & $-0,56$ & 0,49 & $-0,38$ & -6 & 1 & 6 & -5 & -9 & -5 & 6 & 5 & 1,0 & $-0,4$ & 0,6 & $-1,2$ & $-1,4$ \\
\hline$\overline{\mathrm{DP}}\left(\mathrm{s}_{\mathrm{ii}}\right)$ & 0,53 & 0,32 & 0,53 & 0,42 & 5 & 5 & 5 & 3 & 5 & 4 & 6 & 4 & 0,3 & 0,3 & 0,3 & 0,4 & 0,4 \\
\hline $\mathrm{DP}\left(\mathrm{s}_{\mathrm{ii}}{ }^{\prime}-\mathrm{s}_{\mathrm{ik}}\right)$ & 0,79 & 0,48 & 0,79 & 0,64 & 8 & 8 & 8 & 5 & 7 & 6 & 9 & 6 & 0,4 & 0,5 & 0,4 & 0,5 & 0,7 \\
\hline $\mathrm{DP}\left(\mathrm{s}_{\mathrm{ii}}{ }^{\prime}-\mathrm{S}_{\mathrm{kk}}\right)$ & 0,73 & 0,45 & 0,73 & 0,59 & 7 & 7 & 7 & 4 & 7 & 6 & 9 & 5 & 0,4 & 0,5 & 0,4 & 0,5 & 0,6 \\
\hline
\end{tabular}

DP $\left(\mathrm{s}_{\mathrm{ii}}\right)$, desvio-padrão das estimativas de capacidade geral de combinação (CEC); DP $\left(\mathrm{s}_{\mathrm{ii}}{ }^{\prime} \mathrm{s}_{\mathrm{ik}}\right)$, contraste da estimativa de CEC entre híbridos, obtidos do cruzamento entre genitores i e genitores i e k; DP $\left(\mathrm{s}_{\mathrm{ii}}-\mathrm{s}_{\mathrm{kk}}\right)$, contraste de estimativas de CEC de híbridos simples, sem a presença de genitores em comum (i, i', k, k'). 
resistentes à helmintosporiose; $\mathrm{LGS}_{4} \mathrm{xLGS}$, resistente à ferrugem-polissora; e $\mathrm{LGS}_{6} \mathrm{xLGS}$ 10, resistente à mancha-branca, em Pitangueiras. Estes resultados indicam a contribuição expressiva da CEC para o desempenho dos cruzamentos, quanto às diferentes características. Dos híbridos citados anteriormente como mais promissores, $\mathrm{LGS}_{3} \mathrm{xLGS}$ e $\mathrm{LGS}_{2} \mathrm{xLGS}$ também apresentaram as maiores médias de $\hat{s}_{\mathrm{ii}}$ quanto à produtividade, considerando-se os quatro locais.

Embora os modelos fixos de dialelos tenham suas observações de estimativas de capacidade geral de combinação $\left(\mathrm{g}_{\mathrm{i}}\right)$ e capacidade específica de combinação $\left(\mathrm{s}_{\mathrm{ii}}\right)$ limitadas ao grupo de genótipos avaliados no dialelo, os componentes quadráticos destes modelos podem expressar a variabilidade genética do material estudado, o que permite realizar inferências sobre o tipo de ação gênica predominante para cada uma das características (Cruz et al., 2004).

Os efeitos significativos de CGC e CEC e as interações com locais indicam que tanto os efeitos aditivos quanto os não aditivos são importantes para as características estudadas. Contudo, as estimativas dos componentes quadráticos de $\operatorname{CGC}\left(\phi_{\mathrm{g}}\right), \operatorname{CEC}\left(\phi_{\mathrm{s}}\right)$ e a razão entre estes $\left(\phi_{\mathrm{g}} / \phi_{\mathrm{s}}\right)$, obtida por meio da esperança do quadrado médio nas análises de variância, indicam que os efeitos gênicos aditivos são os mais importantes para as características altura de espiga e resistência à mancha-branca. Os resultados obtidos no presente trabalho concordam com os de Paterniani et al. (2000), Carson (2001), Pegoraro et al. (2002), Silva \& Moro (2004), Derera et al. (2007), Lopes et al. (2007) e Vivek et al. (2010), que relatam que a ação gênica aditiva tem maior importância do que a dominante, para a resistência à mancha-branca. Contudo, Das et al. (1989a, 1989b) e Amaral (2005) verificaram que os efeitos dominantes são mais importantes que os aditivos, para a resistência à mancha-branca.

Para resistência a $E$. turcicum, $P$. zeae e $P$. polysora, os compontes quadráticos obtidos mostram que os efeitos dominantes foram mais importantes, o que difere das respostas encontradas por Carson (1995), Paterniani et al. (2000) e Vivek et al. (2010).

\section{Conclusões}

1. Os efeitos gênicos aditivos são mais importantes para as características altura de espiga e resistência a Phaeosphaeria maydis, e os efeitos não aditivos são mais importantes para produtividade, altura de planta e resistência a Exserohilum turcicum, Physopella zeae e Puccinia polysora.

2. As linhagens endogâmicas $\mathrm{LGS}_{2}, \mathrm{LGS}_{9}, \mathrm{LGS}_{4}$ e $\mathrm{LGS}_{3}$ são as que participam dos cruzamentos com maior potencial de produtividade e resistência a doenças foliares.

3. Os híbridos resultantes dos cruzamentos $\mathrm{LGS}_{3} \mathrm{xLGS}$, $\mathrm{LGS}_{2} \mathrm{xLGS}, \mathrm{LGS}_{2} \mathrm{xLGS} \mathrm{L}_{4}$ e $\mathrm{LGS}_{2} \mathrm{xLGS}$ têm maior resistência a doenças foliares e apresentam maior produtividade.

\section{Agradecimentos}

Ao Programa de Mestrado em Genética e Biologia Molecular, da Universidade Estadual de Londrina, e à Empresa GS Pesquisa, pelo apoio à realização deste trabalho.

\section{Referências}

AMARAL, A.L. do. Etiologia e genética da resistência à mancha branca do milho. 2005. 93p. Tese (Doutorado) - Universidade Federal do Rio Grande do Sul, Porto Alegre.

CARSON, M.L. A new gene in maize conferring the "chlorotic halo" reaction to infection by Exserohilum turcicum. Plant Disease, v.79, p.717-720, 1995.

CARSON, M.L. Inheritance of resistance to Phaeosphaeria leaf spot of maize. Plant Disease, v.85, p.798-800, 2001.

CRUZ, C.D. Programa Genes: aplicativo computacional em genética e estatística. Viçosa: UFV, 2001. 442p.

CRUZ, C.D.; REGAZZI, A.J.; CARNEIRO, P.C.S. Modelos biométricos aplicados ao melhoramento genético. 3.ed. Viçosa: UFV, 2004. 480p.

DAS, S.N.; PRODHAN, H.S.; KAISER, S.A.K.M. Further studies on the inheritance of resistance to Phaeosphaeria leaf spot of maize. Indian Journal of Mycological Research, v.27, p.127-130, 1989a.

DAS, S.N.; SINHAMAHAPATRA, S.P.; BASAK, S.L. Inheritance of resistance to Phaeosphaeria lef spot maize. Annual Agricultural Research of Nadia, v.10, p.182-184, 1989b.

DERERA, J.; TONGOONA, P.; VIVEK, B.S.; VAN RIJ, N.; LAING, M.D. Gene action determining Phaeosphaeria leaf spot disease resistance in experimental maize hybrids. South African Journal of Plant and Soil, v.24, p.138-144, 2007.

EMYGDIO, B.M.; IGNACZAK, J.C.; CARGNELUTTI FILHO, A. Potencial de rendimento de grãos de híbridos comerciais simples, triplos e duplos de milho. Revista Brasileira de Milho e Sorgo, v.6, p.95-103, 2007.

FORSTHOFER, E.L.; SILVA, P.R.F. da; STRIEDER, M.L.; MINETTO, T.; RAMBO, L.; ARGENTA, G.; SANGOI, L.; SUHRE, E.; SILVA, A.A. Desempenho agronômico e econômico 
do milho em diferentes sistemas de manejo e épocas de semeadura. Pesquisa Agropecuária Brasileira, v.41, p.399-407, 2006.

GRIFFING, B. Concept of general and specific combining ability in relation to diallel crossing systems. Australian Journal of Biological Sciences, v.9, p.463-493, 1956.

GUIA Agroceres de sanidade. São Paulo: Sementes Agroceres, 1996. 72p.

GUIMARÃES, P. de S.; PATERNIANI, M.E.A.G.Z.; DUDIENAS, C.; LÜDERS, R.R.; GALLO, P.B. Capacidade combinatória para resistência à mancha branca em linhagens endogâmicas de milho. Summa Phytopathologica, v.35, p.282-287, 2009.

LOPES, M.T.G.; LOPES, R.; BRUNELLI, K.R.; SILVA, H.P. da; MATIELlO, R.R.; CAMARGO, L.E.A. Controle genético da resistência à mancha-de-Phaeosphaeria em milho. Ciência Rural, v.37, p.605-611, 2007.

MIRANDAFILHO, J.B. de; VENCOVSKY, R. Analysis of variance with interaction of effects. Revista Brasileira de Genética, v.18, p.129-134, 1995.

PATERNIANI, M.E.A.G.Z.; SAWAZAKI, E.; DUDIENAS, C.; DUARTE, A.P.; GALLO P.B. Diallel crosses among maize lines with emphasis on resistance to foliar diseases. Genetics and Molecular Biology, v.23, p.381-385, 2000.

PEGORARO, D.G.; BARBOSA NETO, J.F.; DAL SOGLIO, F.K; VACARO, E.; NUSS, C.N.; CONCEIÇÃO, L.D.H. Herança da resistência à mancha foliar de feosféria em milho. Pesquisa Agropecuária Brasileira, v.37, p.329-336, 2002.
PINTO, N.F.J. de A. Controle químico de doenças foliares em milho. Revista Brasileira de Milho e Sorgo, v.3, p.134-138, 2004.

SAS INSTITUTE. Getting started with the SAS learning edition. Cary: SAS Institute, 2002. 200p.

SILVA, H.P. da; BARBOSA, M.P.M.; NASS, L.L.; CAMARGO, L.E.A. Capacidade de combinação e heterose para resistência a Puccinia polysora Underw. em milho. Scientia Agricola, v.58, p.777-783, 2001.

SILVA, H.P. da; MORO, J.R. Diallel analysis of maize resistance to Phaeosphaeria maydis. Scientia Agricola, v.61, p.36-42, 2004.

SILVA, R.G.; GALVÃO, J.C.C.; MIRANDA, G.V.; OLIVEIRA, E. de. Controle genético da resistência aos enfezamentos do milho. Pesquisa Agropecuária Brasileira, v.38, p.921-928, 2003.

SILVEIRA, F.T.; JUNQUEIRA, B.G.; SILVA, P.C. da; MORO, J.R. Comportamento de linhagens elites de milho para resistência aos enfezamentos. Revista Brasileira de Milho e Sorgo, v.5, p.431-442, 2006.

VIVEK, B.; ODONGO, O.; NJUGUNA, J.; IMANYWOHA, J.; BIGIRWA, G.; DIALLO, A.; PIXLEY, K. Diallel analysis of grain yield and resistance to seven diseases of African maize (Zea Mays L.) inbred lines. Euphytica, v.172, p.329-340, 2010.

VON PINHO, R.G.; RAMALHO, M.A.P.; RESENDE, I.C.; SILVA, H.P.; POZAR, G. Reação de híbridos comerciais de milho às ferrugens polissora e tropical. Pesquisa Agropecuária Brasileira, v.36, p.439-445, 2001.

Recebido em 26 de agosto de 2011 e aprovado em 16 de fevereiro de 2012 